\title{
LITERACIA DE INFORMAÇÃO NO ENSINO MÉDIO: Referencial Para Pesquisa de Informação Científica
}

\author{
Fatima Passos Kanitar ${ }^{1}$ \\ Rosália Maria Duarte ${ }^{2}$
}

\begin{abstract}
RESUMO
Essa pesquisa faz parte de investigações sobre o tema Literacia de Informação, que abrangem as competências de pesquisa, seleção e tratamento de informação científica com recurso às Tecnologias de Informação e Comunicação. Em razão do problema apresentado por outras pesquisas de que estudantes do Ensino Superior não possuem estas competências, o trabalho aqui apresentado considera que os mesmos devem ingressar num curso de Graduação com conhecimento desta matéria. Destaca-se a importância do uso de elementos de referência para o desenvolvimento da Literacia de Informação. Neste seguimento, este artigo tem como objetivo mostrar as alterações realizadas no referencial de avaliação dessas competências junto a estudantes do Ensino Superior da área de Educação, com vistas à formação de estudantes do Ensino Médio Técnico da área de mecânica e o seu uso nesse contexto. O estudo indicou a necessidade de adaptar o referencial, sobretudo os exemplos, para atender aos estudantes da área de mecânica.
\end{abstract}

Palavras-chave: Literacia de informação. Ensino Médio. Competências. Referencial de avaliação.

\section{INFORMATION LITERACY IN SECONDARY SCHOOL: BENCHMARK FOR ASSESSING SCIENTIFIC INFORMATION}

\section{ABSTRACT}

That research is part of investigations on the theme Information Literacy, that includes the competences to research, select and process scientific information using Information and Communication Technologies. Due to results of other researches indicate that undergraduate students do not have these competences, in this research is considered that students should apply for undergraduate course with this knowledge. It is highlights the importance in the use of reference elements for Information Literacy progress. In this sequence, that paper has as objective to show the alterations accomplished in the benchmark for assessing the competences of undergraduate students, of the Education area, aiming to the learning of Technical Secondary School students, of the mechanical area, and the application in this context. The study suggested necessity to suit the benchmark, especially, the examples, to attend the students of mechanical area.

Keywords: Information literacy. Secondary School. Competences. Benchmark.

Recebido em: $25 / 8 / 2018$

Aceito em: 29/6/2019

\footnotetext{
${ }^{1}$ Pós-doutorado em Educação pela Pontifícia Universidade Católica do Rio de Janeiro - PUC/RJ (2018). Doutorado em Multimídia em Educação pela Universidade de Aveiro (Portugal, 2014). Mestrado em Tecnologia pelo Centro Federal de Educação Tecnológica Celso Suckow da Fonseca (2005). Especialização em Docência pela Faculdade Bithencourt da Silva (1997). Licenciatura em Engenharia Mecânica pela Fundação Técnico Educacional Souza Marques (1981). Professora do Centro Federal de Educação Tecnológica Celso Suckow da Fonseca. http:// lattes.cnpq.br/0713172575166560. https://orcid.org/0000-0002-4890-9032. fkanitar@yahoo.com.br.

2 Graduação em Psicologia pela Universidade do Estado do Rio de Janeiro (1984). Mestrado em Educação pela Fundação Getúlio Vargas - RJ (1991). Doutorado em Educação pela Pontifícia Universidade Católica do Rio de Janeiro (2000). Professora associada da PUC-Rio, onde coordena o Grupo de Pesquisa Educação e Mídia, vinculado ao PPGE. Membro da Associação Nacional de Pós-Graduação e Pesquisa em Educação, da Associação Brasileira de Pesquisadores e Profissionais de Educomunicação e do Grupo de Trabalho Children, Youth and Media da European Communication Research and Education Associantion (Ecrea). Editora da Revista Educação On-line (Educação PUC-Rio), membro do Conselho Editorial da Revista Educação \& Realidade (UFRGS), da Revista Education Review e do Conselho Editorial da Revista InterScientia. Membro fundador da Rede Kino - Rede Latino-Americana de Cinema, Educação e Audiovisual. http://lattes.cnpq. br/8252075995504939. https://orcid.org/0000-0002-5758-2529.rosalia@puc-rio.br
} 
Este artigo parte do problema apontado por outros estudos de que a maioria dos estudantes, ao ingressarem numa universidade, não demonstram ações que comprovem possuir competências de pesquisa, seleção e tratamento de informação científica, com recurso às Tecnologias de Informação e Comunicação (TICs). Os estudantes ficam mobilizados com a quantidade de informações oferecidas pela internet e não sabem identificar as que podem utilizar em seus trabalhos científicos, por não saberem distinguir se um determinado autor é confiável, se a informação é ou não fidedigna, se existe imparcialidade, e como arquivá-la para posterior recuperação e uso.

Este estudo considera que a aprendizagem da matéria de Literacia de Informação (LI), relacionada com as competências mencionadas, deve ser considerada no Ensino Médio (EM), com o intuito de os estudantes ingressarem numa universidade sem estas dificuldades.

Assim, este texto tem como objetivo mostrar as alterações feitas no Referencial de Avaliação dessas competências, desenvolvido num estudo a nível de Pós-Graduação em Educação numa universidade portuguesa, para atender a formação em LI de estudantes do Ensino Médio Técnico da área de mecânica, seu uso e contexto.

\section{REFERENCIAL TEÓRICO}

A adoção de um Referencial de Avaliação para a aprendizagem de LI no contexto do EM fundamenta-se na necessidade de formação nesta matéria apontada por diversos autores de renome, tanto do ponto de vista de se ter uma base para a aprendizagem e avaliação desta matéria, com critérios bem-definidos, quanto da sua inclusão no currículo.

Não se pretende fazer uma recensão bibliográfica que esgote o tema, ou relatar o estado da arte atual, mas, a partir do conteúdo do referencial teórico apresentado, viabilizar a análise e discussão dos resultados.

\section{Dificuldade dos Estudantes}

Como já mencionado, o texto apresentado parte do problema de que a maioria dos estudantes do EM desconhecem o significado de LI e não reconhecem sites fidedignos, apresentando dificuldades em identificar bases de dados de informação científica, o seu significado e os repositórios científicos, somadas à dificuldade de identificar autores e periódicos de renome, devendo apresentar estas competências relacionadas com a LI ao ingressarem numa universidade (BUNDY, 2004; JOVIC, 2016; MARTIN; GARCIA; MCPHEE, 2012; SORMUNEN; TANNI; HEINSTRÖM, 2013).

\section{Contexto dos Estudantes Relacionado ao uso das TICs}

Não se pode descartar que a facilidade propiciada pela internet no acesso às informações está diretamente ligada aos recursos das TICs, o que pode acarretar sobrecarga de informações, confiáveis ou não, derivando no aumento da dificuldade para gerenciá-las, gerando plágio. 
Diante do uso diário de computadores portáteis, smartphones e tablets, por parte dos alunos do EM, os estudantes desse nível de ensino, conforme Jovic (2016), supõem ter as competências para encontrar e avaliar a informação necessária para suas pesquisas. Cabe assinalar que saber utilizar a internet não significa necessariamente ter competências relacionadas com a Ll e que estas não são intuitivas; elas precisam ser ensinadas.

Visando a responder às demandas atuais para atuarem em ambientes de colaboração pelo uso de uma wiki, google docs ou para compartilhar informação em "nuvem" (google drive, dropbox, etc.), julga-se de toda importância considerar -Ensino e Aprendizagem (E/A) de competências que desenvolvam a capacidade de selecionar e avaliar a confiabilidade de informações, o pensamento crítico, o aprender a aprender e o saber mobilizar o conhecimento adquirido no ambiente escolar e profissional.

Essa necessidade é apontada em outros estudos sobre a inclusão desta matéria no currículo do EM (MARTIN; GARCIA; MCPHEE, 2012; PINTO; PONJUÁN; URIBE-TIRADO, 2015; SALISBURY; CORBIN; PESETA, 2013; SORMUNEN; TANNI; HEINSTRÖM, 2013). Neste artigo estas competências estão relacionadas com pesquisa, seleção e tratamento de informação científica com recurso às TICs, denominadas -Literacia de Informação (LI), no contexto do -Ensino Médio (- EM).

\section{Referencial de Avaliação}

Para o E/A dessas competências é necessário ter uma base que fundamente o seu progresso, sendo evidenciado na ementa da disciplina sobre essa literacia. Para tal, é necessário que se tenha um conjunto de elementos como referência a ser seguida. Com vista à sua construção, realizou-se a adaptação de um Referencial de Avaliação destas competências, desenvolvido e validado em investigação realizada junto a estudantes do primeiro ano letivo dos cursos de Mestrado e Doutorado do Departamento de Educação da Universidade de Aveiro (UA). Esse Referencial de Avaliação teve como base o trabalho de Timmers e Glas (2010) e Lopes e Pinto (2010), fundamentado em contributos de bibliotecários da UA e a literatura do campo (LOPES; PINTO, 2010). Sua confiabilidade foi estabelecida pelos contributos de docentes/bibliotecários da área da educação e das TICs de Instituições de Ensino Superior (IESs) de Portugal e do Brasil. Foi enviado um questionário para estes profissionais para se obter indicações e comentários do grau de relevância atribuído para cada indicador das dimensões - competências relacionadas com a LI -do Referencial de Avaliação. Desse modo, obteve-se os devidos critérios (referente) e indicadores (referido), na acepção de Hadji (1994) e Figari (1994). Segundo os autores, a definição dos critérios e indicadores é essencial para emitir juízos de valor com relação às competências em LI.

Considerando a necessidade de que os estudantes do EM estejam também preparados para as demandas que enfrentarão na realização de suas pesquisas, e visando aàinclusão dessa literacia em uma escola de Ensino Médio Técnico da cidade do Rio de Janeiro, foram ministrados cursos de extensão para estudantes da terceira série do curso técnico de mecânica, tendo como base o Referencial de Avaliação produzido no âmbito do Ensino (ES) na UA. Desse modo, foram produzidas as adaptações necessárias no Referencial de Avaliação das competências do ES (na área de educação) para o EM (na área de mecânica), visando o E/A da competência de pesquisa de informação científica com recurso às TICs, utilizando-se exemplos da área de mecânica. 


\section{OBJETIVO}

Ante o exposto, este artigo tem como objetivo:

- Apresentar as alterações e utilização do Referencial de Avaliação.

- Discutir as referidas alterações do Referencial de Avaliação no contexto supramencionado.

Na próxima seção apresenta-se a definição dos termos de Competências e Literacia de Informação tomados como referência. Seguidamente, as alterações realizadas no Referencial de Avaliação são mostradas, analisadas e discutidas. Finalmente, são evidenciados os resultados do trabalho desenvolvido junto aos estudantes do Ensino Médio Técnico, com as devidas recomendações.

\section{COMPETÊNCIAS RELACIONADAS COM A LITERACIA DE INFORMAÇÃO}

$\mathrm{Na}$ área da educação são considerados alguns modelos conceituais para a definição do termo competência: cognitiva, operacional ou funcional e transversal. A competência cognitiva implica conhecimento não observável, opondo-se à competência operacional ou funcional, que envolve saber realizar tarefas específicas; a competência transversal é considerada o saber colocar o conhecimento em ação sem haver conteúdos formais a seguir, ou seja, o "saber mobilizar" conhecimentos previamente adquiridos, em situações complexas e imprevisíveis.

O estudo a que se refere este artigo utiliza o termo competência referindo-se a saber mobilizar, combinar/integrar e transpor recursos individuais incorporados (conhecimento, capacidade e atitudes) em quaisquer ambientes para a resolução de famílias de problemas (LE BOTERF, 2005).

\section{Literacia de Informação}

Não existe um consenso por parte de autores para a definição do termo Literacia de Informação. Para aprofundar o entendimento dessa terminologia, fez-se necessário fazer um levantamento comparativo do modo como o termo é utilizado em estudos realizados nesse campo.

Quadro 1 - Definições do termo Literacia de Informação

\begin{tabular}{|l|l|}
\hline \multicolumn{2}{|c|}{ Definições de Literacia de Informação } \\
\hline \multicolumn{1}{|c|}{ Autor/Ano } & \multicolumn{1}{c|}{ Definição } \\
\hline JOVIC, 2016 & $\begin{array}{l}\text { Reconhecer a necessidade de informação científica, sabendo pesquisar, } \\
\text { selecionar, tratar e usar a informação, com recurso às TIC. }\end{array}$ \\
\hline $\begin{array}{l}\text { SORMUNEN; TANNI; } \\
\text { HEINSTRÖM, 2013 }\end{array}$ & $\begin{array}{l}\text { Pesquisar e recuperar a informação científica, de forma colaborativa e } \\
\text { cooperativa. }\end{array}$ \\
\hline ETS, 2006 & $\begin{array}{l}\text { Definir um tópico de pesquisa, acessar, avaliar, gerenciar, integrar, criar e } \\
\text { comunicar a informação científica. }\end{array}$ \\
\hline BUNDY, 2004 & $\begin{array}{l}\text { Competências que representam: i) o reconhecimento da necessidade de } \\
\text { informação e a determinação da sua natureza e da sua extensão; ii) o encontro } \\
\text { de forma eficaz da informação necessária; iii) a avaliação crítica da informação } \\
\text { e do processo de pesquisa; iv) o tratamento da informação recuperada; v) o uso } \\
\text { da informação para construir novos conhecimentos; e, vi) o uso da informação } \\
\text { respeitando questões éticas, econômicas, legais e sociais. }\end{array}$ \\
\hline $\begin{array}{l}\text { EISENBERG; JOHNSON; } \\
\text { BERKOWITZ, 2010 }\end{array}$ & $\begin{array}{l}\text { Saber: i) definir uma tarefa; ii) determinar estratégias de pesquisa de informação; } \\
\text { iii) localizar e acessar a informação; e iv) sintetizar e avaliar a tarefa realizada. }\end{array}$ \\
\hline
\end{tabular}


No Quadro apresentado percebe-se que o termo Literacia de Informação tem sido definido como competência para saber identificar uma necessidade de informação científica, localizar, avaliar e usar a informação pesquisada, gerando um conteúdo novo. Nesse contexto, o estudante deve ser crítico, sabendo aprender a aprender, o que conduz para a aprendizagem ao longo da vida.

A partir das definições mencionadas, no estudo que originou este artigo o termo Literacia de Informação é definido como as competências de pesquisa, seleção e tratamento de infomação científica, com recurso às TICs.

\section{ALTERAÇÕES DO REFERENCIAL DE AVALIAÇÃO}

O Referencial de Avaliação ocupa-se de tarefas "em ação", o que vai ao encontro do conceito de competência considerado neste trabalho. Nesse segmento, o curso de competências referentes à pesquisa de informação científica, com recurso às TICs, foi ministrado envolvendo a ação de estudantes em sua área de estudo. Para tal, foram feitas as alterações mostradas nos Quadros a seguir. São utilizadas as siglas ES para Ensino Superior e EM para Ensino Médio. A competência de pesquisa é sistematizada por um conjunto de 5 critérios e 23 indicadores. Cada um dos critérios será apresentado num Quadro.

Quadro 2 - Critério C1 da competência de pesquisa de informação científica

\begin{tabular}{|c|c|c|c|}
\hline \multicolumn{4}{|c|}{$\begin{array}{l}\text { Referencial de avaliação referente às competências de pesquisa de informação científica, } \\
\text { com recurso às TIC }\end{array}$} \\
\hline Ensino & Critérios & Indicadores & Exemplos \\
\hline ES & \multirow{6}{*}{$\begin{array}{l}\text { C1. Definir } \\
\text { com profi- } \\
\text { ciência uma } \\
\text { necessidade } \\
\text { de informa- } \\
\text { ção científica. }\end{array}$} & \multirow{2}{*}{$\begin{array}{l}\text { I1. Identificar uma ne- } \\
\text { cessidade de informação } \\
\text { científica, que pode partir } \\
\text { de um problema relacio- } \\
\text { nado com os processos } \\
\text { de ensino e aprendizagem } \\
\text { ou definido a partir da } \\
\text { consulta da literatura da } \\
\text { especialidade. }\end{array}$} & $\begin{array}{l}\text { Vários autores reportam que os alunos do Ensino Superior } \\
\text { (ES) têm falta de competências relacionadas com a LI em } \\
\text { recursos digitais. Assim, a título de exemplo, no caso da } \\
\text { presente investigação, a necessidade de informação pren- } \\
\text { de-se com as competências relacionadas com a LI e como } \\
\text { estas têm sido avaliadas. }\end{array}$ \\
\hline EM & & & $\begin{array}{l}\text { A necessidade de informação pode prender-se, por exem- } \\
\text { plo, a otimização de processo na produção de estruturas } \\
\text { primárias de carrocerias de veículos de transporte público. }\end{array}$ \\
\hline ES & & \multirow{2}{*}{$\begin{array}{l}\text { 12. Definir os tópicos que } \\
\text { se articulam com a ne- } \\
\text { cessidade de informação } \\
\text { científica identificada. }\end{array}$} & $\begin{array}{l}\text { Tendo em conta a necessidade de informação relativa à } \\
\text { avaliação de competências relacionadas com a LI, dois } \\
\text { tópicos relacionados são o conceito de } \mathrm{LI} \text { e competências } \\
\text { relacionadas com a } \mathrm{LI} \text { em recursos digitais }\end{array}$ \\
\hline EM & & & $\begin{array}{l}\text { Tendo em conta a necessidade de informação relativa à oti- } \\
\text { mização de processo na produção de estruturas primárias } \\
\text { de carrocerias de veículos de transporte público, dois tópi- } \\
\text { cos relacionados são a seleção dos materiais e das variáveis } \\
\text { das operações para a fabricação da estrutura principal de } \\
\text { um ônibus. }\end{array}$ \\
\hline ES & & \multirow{2}{*}{$\begin{array}{l}\text { I3. Definir questões/ } \\
\text { objetivos de investigação } \\
\text { a partir de um problema } \\
\text { (que implique necessida- } \\
\text { de de informação científi- } \\
\text { ca) ou de uma revisão de } \\
\text { literatura de qualidade. }\end{array}$} & $\begin{array}{l}\text { Um dos objetivos da presente investigação é analisar os } \\
\text { conceitos de LI e de competências relacionadas com a LI } \\
\text { com recurso às TIC, explorados na literatura da especiali- } \\
\text { dade. }\end{array}$ \\
\hline EM & & & $\begin{array}{l}\text { Dois dos objetivos da investigação são caracterizar os parâ- } \\
\text { metros dos materiais e simular as tecnologias de produção. }\end{array}$ \\
\hline
\end{tabular}

De acordo com o Quadro 2, na área de mecânica foi identificada como necessidade de informação científica a otimização de processo na produção de estruturas primárias de carrocerias de veículos de transporte público, no indicador I1. Tendo em conta a premência de obtenção de carrocerias de materiais mais leves, resistentes, com a capacidade de 
moldar e adaptar estruturas com aerodinâmica mais eficiente. No caso do 12, os tópicos relacionados com a necessidade de informação são a seleção dos materiais e das variáveis das operações para a fabricação da estrutura principal de um ônibus. Para o 13 são citados, como exemplos, para os objetivos da investigação, caracterizar os parâmetros dos materiais e simular as tecnologias de produção (GONZALEZ LOZANO et al., 2016; LOPES; GÜRDAL; CAMANHO, 2008). Neste critério houve somente alterações nos exemplos.

Quadro 3 - Critério C2 da competência de pesquisa de informação científica

\begin{tabular}{|c|c|c|c|}
\hline \multicolumn{4}{|c|}{ Referencial de avaliação referente às competências de pesquisa de informação científica, com recurso às TIC } \\
\hline Ensino & Critérios & Indicadores & Exemplos \\
\hline ES & \multirow{15}{*}{$\begin{array}{l}\text { C2. Construir } \\
\text { uma estratégia } \\
\text { de pesquisa de } \\
\text { in forma çã o } \\
\text { científica ri- } \\
\text { gorosa, des- } \\
\text { crevendo as } \\
\text { etapas. }\end{array}$} & \multirow[t]{2}{*}{$\begin{array}{l}\text { 14. Definir as estratégias } \\
\text { de pesquisa a explorar. }\end{array}$} & \multirow{2}{*}{$\begin{array}{l}\text { As etapas de pesquisa da informação científica passam por identi- } \\
\text { ficar o problema e os tópicos e questões relacionadas, as palavras- } \\
\text {-chave e termos similares, definir as bases de dados em que será } \\
\text { efetuada a pesquisa... }\end{array}$} \\
\hline EM & & & \\
\hline ES & & \multirow{3}{*}{\begin{tabular}{|l|} 
15. Estabelecer palavras- \\
-chave a partir da temá- \\
tica a estudar (problema, \\
questões... ou de pala- \\
vras-chave encontradas \\
em fontes de referência), \\
preferencialmente em \\
várias línguas, para uma \\
maior abrangência de \\
resultados.
\end{tabular}} & $\begin{array}{l}\text { Para o tema desta investigação - competências relacionadas com a } \\
\text { LI com recurso às TIC de alunos do ES: information seeking, informa- } \\
\text { tion seeking behaviour, information literacy, ICT skills, assessment, } \\
\text { higher education... }\end{array}$ \\
\hline & & & \\
\hline EM & & & $\begin{array}{l}\text { Para o tema de investigação - produção de estruturas primárias de } \\
\text { carrocerias de veículos de transporte público: filament winding, fiber } \\
\text { placement, public transport ... }\end{array}$ \\
\hline ES & & \multirow{2}{*}{$\begin{array}{l}\text { 16. Identificar outros } \\
\text { termos relacionados } \\
\text { com as palavras-chave a } \\
\text { empregar na pesquisa, } \\
\text { os descritores, usando as } \\
\text { potencialidades de ferra- } \\
\text { mentas on-line. }\end{array}$} & $\begin{array}{l}\text { Pesquisar outras palavras-chave no Thesaurus da base de dados da } \\
\text { ERIC (http://www.eric.ed.gov/ERICWebPortal/thesaurus/thesaurus. } \\
\text { jsp). Por exemplo, para a palavra skill: competence; para higher } \\
\text { education: graduate study, doctoral education; e para information } \\
\text { seeking: online searching, library search. Podem ainda ser usados } \\
\text { termos integrados em ferramentas da Web 2.0, como o TEL Thesau- } \\
\text { rus and Dictionary (http://thesaurus.telearn.org/The_TEL_Thesau- } \\
\text { rus_and_Dictionary_metaproject). }\end{array}$ \\
\hline EM & & & $\begin{array}{l}\text { Pesquisar outras palavras-chave em ferramentas da Web 2.0, como } \\
\text { o Thesaurus and Dictionary (https://www2.powerthesaurus.org/); } \\
\text { por exemplo, para a palavra placement: placing; para fiber: filament; } \\
\text { para public: urban; e para transport: transportation, transporting. }\end{array}$ \\
\hline ES & & \multirow{2}{*}{$\begin{array}{l}\text { 17. Prever a possibilidade } \\
\text { de estreitar/adaptar o } \\
\text { foco de pesquisa de for- } \\
\text { ma a obter um número } \\
\text { de fontes variado, rele- } \\
\text { vante, útil... mas simulta- } \\
\text { neamente analisável em } \\
\text { tempo profícuo. }\end{array}$} & \multirow{2}{*}{$\begin{array}{l}\text { Delimitando o período de tempo ou o tipo de fontes ou adicionando } \\
\text { outras palavras-chave... }\end{array}$} \\
\hline EM & & & \\
\hline ES & & \multirow{2}{*}{$\begin{array}{l}\text { 18. Identificar as fontes } \\
\text { de pesquisa de infor- } \\
\text { mação científica que } \\
\text { serão necessárias para a } \\
\text { pesquisa. }\end{array}$} & \multirow[t]{2}{*}{$\begin{array}{l}\text { Como bases de dados acadêmicas, editoras de revistas científicas, } \\
\text { repositórios abertos, agregadores de bibliotecas... }\end{array}$} \\
\hline EM & & & \\
\hline ES & & \multirow{2}{*}{$\begin{array}{l}\text { 19. Caracterizar fontes } \\
\text { primárias e fontes secun- } \\
\text { dárias. }\end{array}$} & \multirow{2}{*}{$\begin{array}{l}\text { Fontes primárias - fontes originais de informação científica ou de } \\
\text { novas ideias sobre um tópico, e as fontes secundárias - fontes que } \\
\text { interpretam, atribuem valor e delineiam conclusões a partir de } \\
\text { fontes primárias. }\end{array}$} \\
\hline EM & & & \\
\hline ES & & \multirow[t]{2}{*}{$\begin{array}{l}\text { 110. Prever outras estra- } \\
\text { tégias de pesquisa caso } \\
\text { seja necessário. }\end{array}$} & $\begin{array}{l}\text { Alterar palavras-chave, fazer pesquisa de informação científica nou- } \\
\text { tras fontes ou com outros formatos (por exemplo vídeos - http:// } \\
\text { www.youtube.com/watch?v=SUsP9ZOhx3U\&feature=youtu.be). }\end{array}$ \\
\hline EM & & & $\begin{array}{l}\text { Alterar palavras-chave, fazer pesquisa de informação científica em } \\
\text { outras fontes ou com outros formatos (por exemplo vídeos - ht- } \\
\text { tps://www.youtube.com/watch?v=cwteFOCBalg). }\end{array}$ \\
\hline
\end{tabular}


Pela leitura do Quadro 3, as estratégias de pesquisa que devem ser exploradas são comuns para qualquer área de estudo, como indicado em 14. Em relação ao 15, foram estabelecidas novas palavras-chave (fiber placement, public transport) para atender à necessidade de informação científica na área investigada. No 16 os descritores pesquisados no Thesaurus foram alterados. Não houve necessidade de alterar os exemplos do 17, do 18 e do 19, pois, para qualquer domínio do conhecimento, o procedimento é similar para estreitar/adaptar o foco de uma pesquisa, bem como para identificar e caracterizar suas fontes. No exemplo do I10, evidenciando a provável necessidade de usar fontes de diversos formatos, como vídeos, foi necessário alterá-lo para a área de mecânica. No C2 foram substituídos somente alguns exemplos.

Quadro 4 - Critério C3 da competência de pesquisa de informação científica

\begin{tabular}{|c|c|c|c|c|}
\hline \multicolumn{5}{|c|}{ Referencial de avaliação referente às competências de pesquisa de informação científica, com recurso às TIC } \\
\hline Ensino & Critérios & & Indicadores & Exemplos \\
\hline ES & \multirow{9}{*}{$\begin{array}{l}\text { ES } \\
\text { C3. Usar } \\
\text { com } \\
\text { proficiência } \\
\text { técnicas } \\
\text { e serviços } \\
\text { de apoio à } \\
\text { pesquisa de } \\
\text { informação } \\
\text { científica } \\
\text { relevantes } \\
\text { para a área } \\
\text { da educa- } \\
\text { ção. }\end{array}$} & \multirow{9}{*}{$\begin{array}{l}\text { EM } \\
\text { C3. Usar com } \\
\text { proficiência } \\
\text { técnicas } \\
\text { e serviços } \\
\text { de apoio à } \\
\text { pesquisa de } \\
\text { informação } \\
\text { científica } \\
\text { relevantes } \\
\text { para a área } \\
\text { em estudo. }\end{array}$} & \multirow{2}{*}{$\begin{array}{l}\text { I11. Explorar bases de dados } \\
\text { com informação científica } \\
\text { integradas nos serviços de } \\
\text { bibliotecas acadêmicas. }\end{array}$} & $\begin{array}{l}\text { Tais como na UA, a B-on, a Academic Search Complete, a ERIC, o RCAAP (disponiveis em: } \\
\text { http://www.ua.pt/sbidm/biblioteca/Pagelmage.aspx?id=8090). }\end{array}$ \\
\hline EM & & & & $\begin{array}{l}\text { Tais como na USP, a Aerospace Database e } a \text { ASTM International (disponiveis em: http:// } \\
\text { www.buscaintegrada.usp.br/primo_library/libweb/action/search.do?dscnt=0\&dstm- } \\
\text { p=1473791964818\&vid=USP\&fromLogin=true ). }\end{array}$ \\
\hline ES & & & \multirow[t]{2}{*}{$\begin{array}{l}\text { I12. Usar catálogos de biblio- } \\
\text { tecas acadêmicas. }\end{array}$} & $\begin{array}{l}\text { Como o disponivel na UA (http://opac.ua.pt/) ou na Universidade de Coimbra (http://webo- } \\
\text { pac.sib.uc.pt/search*por S25). }\end{array}$ \\
\hline EM & & & & $\begin{array}{l}\text { Como o disponível na USP (http://www.sibi.usp.br/), na Universidade de Aveiro (http://opac. } \\
\text { ua.pt/ ) ou na Universidade de Coimbra (http://webopac.sib.uc.pt/search S9). }\end{array}$ \\
\hline ES & & & \multirow[t]{2}{*}{$\begin{array}{l}\text { 113. Utilizar os serviços/recur- } \\
\text { sos de apoio à pesquisa de in- } \\
\text { formação científica facultados } \\
\text { por bibliotecas acadêmicas. }\end{array}$} & $\begin{array}{l}\text { No caso da UA, obter apoio e orientação na pesquisa de informação científica, localizar uma } \\
\text { revista científica (por exemplo, consultando materiais de apoio de bibliotecas acadêmicas - } \\
\text { http://www.ua.pt/sbidm/biblioteca/Pagelmage.aspx?id=8262 ou http://www.lib.jmu.edu/ } \\
\text { resources/subject.aspx?s=32). }\end{array}$ \\
\hline EM & & & & $\begin{array}{l}\text { Consultando materiais de apoio de bibliotecas acadêmicas, no caso da UFRJ (http://www. } \\
\text { coppead.ufrj.br/pt-br/docentes-e-pesquisa/biblioteca/tutoriais/), da UFMG (http://biblio- } \\
\text { teca.qui.ufmg.br/?q=node/18) e da UA (http://www.ua.pt/sbidm/biblioteca/PageText. } \\
\text { aspx?id=8492 ) para localizar uma revista científica, obter apoio e orientação na pesquisa de } \\
\text { informação cientifica. }\end{array}$ \\
\hline ES & & & \multirow{2}{*}{$\begin{array}{l}\text { I14. Usar os operadores de } \\
\text { pesquisa (operadores boolia- } \\
\text { nos, parêntese, truncatura ou } \\
\text { aspas) para alargar ou reduzir } \\
\text { o âmbito da pesquisa de } \\
\text { informação científica. }\end{array}$} & $\begin{array}{l}\text { Como o OR para alargar: "information skill" OR "information literacy"; e para restringir: AND } \\
\text { "higher education" e NOT "secondary education". }\end{array}$ \\
\hline EM & & & & $\begin{array}{l}\text { Como o OR para alargar: "fiber placement" OR "filament winding"; e para restringir: AND } \\
\text { "public transport "e NOT "public transport stop". }\end{array}$ \\
\hline ES & & & \multirow{2}{*}{$\begin{array}{l}\text { 115. Restringir a pesquisa } \\
\text { usando as potencialidades de } \\
\text { pesquisa avançada das ferra- } \\
\text { mentas de bases de dados de } \\
\text { pesquisa. }\end{array}$} & $\begin{array}{l}\text { No RCAAP (http://www.rcaap.pt/search.jsp) pode cruzar-se palavras-chave no título, assunto } \\
\text { ou texto integral, com o nome do autor, definir o tipo de documento (artigo, tese...), etc. }\end{array}$ \\
\hline EM & & & & $\begin{array}{l}\text { No CEFET/RJ (http://biblioteca.cefet-rj.br/) e no RCAAP (https://www.rcaap.pt/search.jsp } \\
\text { ) pode cruzar-se palavras-chave no título, assunto ou texto integral, com o nome do autor, } \\
\text { definir o tipo de documento (artigo, tese...), etc. }\end{array}$ \\
\hline ES & & & \multirow[t]{2}{*}{$\begin{array}{l}\text { I16. Identificar na área de inte- } \\
\text { resse, os autores, as revistas, } \\
\text { os encontros... com maior } \\
\text { reputação/fator de impacto. }\end{array}$} & $\begin{array}{l}\text { Obter a partir da Scimago as revistas da área de educação com maior fator de impacto. } \\
\text { Disponivel em: http://www.scimagojr.com/journalrank.php?area=3300\&category=3304\&coun- } \\
\text { try=all\&year=2011\&order=sj\&\&min=0\&min_type=cd. }\end{array}$ \\
\hline EM & & & & $\begin{array}{l}\text { Obter a partir da Plataforma Sucupira consultas do fator de impacto Capes - Qualis de pe- } \\
\text { rí́dicos. Disponível em: em https://sucupira.capes.gov.br/sucupira/public/consultas/coleta/ } \\
\text { veiculoPublicacaoQualis/listaConsultaGeralPeriodicos.jsf } \\
\text { Obter a partir da Scimago as revistas da área de engenharia mecânica com maior fator de im- } \\
\text { pacto, em http://www.scimagojr.com/journalrank.php?area=2200\&year=2011\&category=2210 }\end{array}$ \\
\hline ES & & & \multirow{2}{*}{$\begin{array}{l}\text { 117. Recuperar informação } \\
\text { científica por intermédio de } \\
\text { alertas de revistas, de e-books } \\
\text { ou de associações (inscrições } \\
\text { on-line). }\end{array}$} & $\begin{array}{l}\text { Fazendo a subscrição de alertas de uma associação ou de uma revista, como o European Jour- } \\
\text { nal of Open, Distance and E-Learning (ver ligação http://www.eurodl.org/?p=subscribe). }\end{array}$ \\
\hline EM & & & & $\begin{array}{l}\text { Fazendo a subscrição de alertas de uma associação ou de uma revista, como o Composite } \\
\text { Structures (ver ligação http://www.journals.elsevier.com/composite-structures ), Composites } \\
\text { Part A: Applied Science and Manufacturing (ver ligação http://www.journals.elsevier.com/ } \\
\text { composites-part-a-applied-science-and-manufacturing) }\end{array}$ \\
\hline ES & & & \multirow{2}{*}{$\begin{array}{l}\text { I18. Cruzar as pesquisas em } \\
\text { diversas fontes de informação } \\
\text { científica, a fim de obter } \\
\text { variedade de pontos de vista } \\
\text { e níveis de cobertura sobre o } \\
\text { tópico estudado. }\end{array}$} & $\begin{array}{l}\text { Como o Google Acadêmico com a biblioteca do conhecimento on-line (b-on) e/ou Eric - inter- } \\
\text { face ebsco. }\end{array}$ \\
\hline EM & & & & $\begin{array}{l}\text { Como o Google Académico (https://scholar.google.com.br/) com a SciELO (http://www.scielo. } \\
\text { br/?lng=pt) e/ou com o SCIRP (http://www.scirp.org/journal/wjm/ ). }\end{array}$ \\
\hline
\end{tabular}


Em concordância com o Quadro 4, sobre técnicas e serviços de apoio à pesquisa, foram mostradas bases de dados da Universidade de São Paulo (USP), ao invés das bases da UA, no indicador I11. No exemplo de I12 foi somada a ligação da USP às das bibliotecas de Aveiro e Coimbra. No I13, para a indicação de materiais de apoio de bibliotecas acadêmicas, julgou-se não considerar os da Universidade de James Madison por serem mais voltados para a área de educação. Acrescentou-se, no entanto, os da Universidade Federal do Rio de Janeiro (UFRJ) e da -Universade Federal de Minas Gerais (UFMG). No caso do uso de operadores de pesquisa, evidenciados no I14, substituiram-se apenas as palavras-chave. Para usar as potencialidades de pesquisa avançada, mostradas no I15, acrescentou-se, para o EM, a ligação da biblioteca do Cefet/RJ. Do mesmo modo, no indicador 116, em adição ao exemplo do SCImago Journal Rank (SJR), evidenciam-se os periódicos de diversas áreas do conhecimento, inclusive engenharia e materiais, com maior reputação segundo a classificação do Qualis, sistema brasileiro de avaliação de periódicos, mantido pela -Coordenação de Aperfeiçoamento de Pessoal de Nível Superior - Capes. No 117 foram mostradas revistas da área de materiais para fazer subscrição. No I18, para cruzar as informações em diversas fontes de informação científica, somado ao Google Acadêmico, foram indicadas a Scientific Electronic Library Online (SciELO) e a Scientific Research Publishing (SCIRP). Foram retiradas a Biblioteca do Conhecimento On-line (b-on) e a Eric. Foi mudado parte do texto de C3, porque é preciso ter esta competência em qualquer área de estudo e não apenas na de educação.

Quadro 5 - Critério C4 da competência de pesquisa de informação científica

\begin{tabular}{|c|c|c|c|}
\hline \multicolumn{4}{|c|}{ Referencial de avaliação referente às competências de pesquisa de informação científica, com recurso às TICs } \\
\hline Ensino & Critérios & Indicadores & Exemplos \\
\hline EM & \multirow{5}{*}{$\begin{array}{l}\text { C4. Pesquisar } \\
\text { com proficiên- } \\
\text { cia literatura } \\
\text { cinzenta confiá- } \\
\text { vel na internet. }\end{array}$} & $\begin{array}{l}\text { I19. Pesquisar teses de Douto- } \\
\text { ramento e/ou dissertações de } \\
\text { Mestrado em repositórios que } \\
\text { sejam fidedignos. }\end{array}$ & $\begin{array}{l}\text { Fazer pesquisa no Repositório Institucional da UA. Disponível } \\
\text { em: http://ria.ua.pt/ ou na Biblioteca Digital de Teses e Disser- } \\
\text { tações da USP em http://www.teses.usp.br/. }\end{array}$ \\
\hline ES & & \multirow[t]{2}{*}{$\begin{array}{l}\text { 120. Pesquisar relatórios em } \\
\text { organizações internacionais de } \\
\text { reconhecido mérito. }\end{array}$} & $\begin{array}{l}\text { Como o relatório sobre educação publicado pela Unesco. Dis- } \\
\text { ponível em: http://www.unesco.org/new/fileadmin/MULTIME- } \\
\text { DIA/HQ/ED/pdf/gmr2011-summary-pt.pdf. }\end{array}$ \\
\hline EM & & & $\begin{array}{l}\text { Como a publicação sobre Biomecânica do movimento humano } \\
\text { publicado pela Unesco e Fundação Vale. Disponível em: http:// } \\
\text { unesdoc.unesco.org/images/0022/002250/225002POR.pdf . }\end{array}$ \\
\hline ES & & $\begin{array}{l}\text { 121. Pesquisar publicações ofi- } \\
\text { ciais em sites de organismos do } \\
\text { governo. }\end{array}$ & $\begin{array}{l}\text { Como as disponibilizadas pelo Ministério da Educação e Ensino } \\
\text { Superior de Portugal. Disponível em: http://www.gepe.minedu. } \\
\text { pt/np4/611.html. }\end{array}$ \\
\hline EM & & $\begin{array}{l}\text { 121. Pesquisar publicações ofi- } \\
\text { ciais em sites de organismos do } \\
\text { governo. }\end{array}$ & $\begin{array}{l}\text { Como as disponibilizadas pelo Ministério da Educação e Cultura } \\
\text { do Brasil em http://www.servicos.gov.br/busca?q=MEC. }\end{array}$ \\
\hline
\end{tabular}

Como visto na Quadro 5, com a finalidade de pesquisar teses e/ou dissertações em repositórios, não houve necessidade de alterar os exemplos da USP e UA no indicador I19. No 120, todavia, considerando o exemplo de pesquisar relatórios em organizações internacionais, foi alterado o da área de educação pelo de Biomecânica, publicado pela Unesco. Foi substituído o exemplo do site português para um brasileiro no 121 . 
Quadro 6-Critério C5 da competência de pesquisa de informação científica

\begin{tabular}{|c|c|c|c|}
\hline Ensino & Critérios & Indicadores & Exemplos \\
\hline ES & \multirow{4}{*}{$\begin{array}{l}\text { C5. Utilizar adequa- } \\
\text { damente outras } \\
\text { fontes de informação } \\
\text { científica digital mais } \\
\text { genéricas. }\end{array}$} & \multirow[t]{2}{*}{$\begin{array}{l}\text { I22. Usar as potencialidades } \\
\text { do Google Acadêmico (http:// } \\
\text { scholar.google.pt/) ou do } \\
\text { Scielo (http://www.scielo.org/ } \\
\text { php/index.php), reconhecendo } \\
\text { as suas limitações. }\end{array}$} & \multirow[t]{2}{*}{$\begin{array}{l}\text { Usar o Google Acadêmico tendo consciência de que os } \\
\text { resultados podem não atender à qualidade da informação. }\end{array}$} \\
\hline EM & & & \\
\hline ES & & \multirow[t]{2}{*}{$\begin{array}{l}\text { 123. Utilizar ferramentas da } \\
\text { Web } 2.0 \text { para a recuperação da } \\
\text { informação científica. }\end{array}$} & $\begin{array}{l}\text { Tais como as acessíveis a partir do Youtube (http://www. } \\
\text { youtube.com/watch?v=rmEzo51e_SQ), do knovio (http:// } \\
\text { go.knovio.com/account/698/link/235)... }\end{array}$ \\
\hline EM & & & $\begin{array}{l}\text { Tais como as acessíveis a partir do Youtube (https://www. } \\
\text { youtube.com/watch?v=FTUw0OWWMLU ), doTED (http:// } \\
\text { www.ted.com/talks/kwabena_boahen_on_a_computer_ } \\
\text { that_works_like_the_brain?language=en\#t-73591)... }\end{array}$ \\
\hline
\end{tabular}

Pela leitura do Quadro 6, não foi necessário modificar o exemplo do I22. Para o indicador 123, entretanto, foi alterado o seu exemplo com a finalidade de atender o contexto de estudo considerado como necessidade de informação científica no curso de mecânica do EM, como a inclusão de um vídeo do TED.

\section{ANÁLISE E DISCUSSÃO DOS RESULTADOS}

Neste texto são evidenciadas a análise e a discussão dos resultados pertinentes à alteração realizada no Referencial de Avaliação com vista a atender o objetivo deste estudo.

\section{Adaptações do Referencial de Avaliação}

No caso do critério $\mathrm{C} 1$, relacionado com a definição de uma necessidade de informação científica, a alteração foi somente nos exemplos de todos os indicadores, 11 ao 13, em razão da necessidade de informação ser de área distinta (ver Quadro 2), o que também é percebido em C2 para os indicadores 15, 16 e 110 para a construção de estratégias de pesquisa, visando à identificação de palavras-chave e de seus termos relacionados, bem como para a consulta de informação em fontes de diversos formatos (ver Quadro 3). De forma contrária, por se tratar de procedimentos de pesquisa comuns para qualquer domínio do conhecimento, foram considerados os mesmos exemplos para os outros indicadores - 14, 17, 18 e 19 de C2. O final do texto do critério C3 (Quadro 4) foi alterado porque o uso das técnicas e serviços de apoio à pesquisa, que se referia somente à área de educação, pode se aplicar a qualquer área de uma pesquisa científica. Foram acrescentados exemplos nos indicadores I12, I13, I15, I16 e I18, mas foi necessário modificar os relacionados aos indicadores I11, I14 e I17, objetivando o escopo do estudo, 0 que, da mesma forma, foi visto para consulta de literatura cinzenta no 120 do critério C4 (Quadro 5). Não houve modificação no exemplo de I19. Foi alterado o exemplo do órgão governamental no indicador 121 . Em relação aos indicadores 122 e 123 de C5 (Quadro 6), tendo em vista o uso de informações mais genéricas, somente o exemplo de 123 foi 
modificado para atender à área de estudo. Assim, levando em conta o exposto relativamente às alterações necessárias no referencial de avaliação, para atender o EM verifica-se que, de maneira geral, poucas foram as modificações nos critérios e indicadores.

O referencial de avaliação foi a base para a apresentação, em formato de slides (Power Point), do curso de extensão do Ensino Médio Técnico sobre essas competências. Os estudantes foram dividos em grupos e proposto um tema, na área de mecânica, para desenvolverem durante o curso. Tiveram de pesquisar considerando os fenômenos estudados na Resistência dos Materiais (por exemplo: tração, cisalhamento, torção, flambagem, flexão e elementos de máquinas) no âmbito da indústria automobilística, aeronáutica, naval, ferroviária e próteses, havendo interação da teoria apresentada com a prática de pesquisa na internet.

Apesar da alteração dos exemplos de alguns indicadores para a área epigrafada, foi necessário esclarecer alguns conceitos, como sinalizados a seguir:

- C1. Definir com proficiência uma necessidade de informação científica

- Diferença entre informação e informação científica.

- C2. Construir uma estratégia de pesquisa de informação científica rigorosa, descrevendo as etapas

- Bases de dados multidisciplinares, como da Capes, Scopus, WEB of Scence, Science Direct, Emerald, Academic Search Complete, e também alguns editores Springer, Taylor and Francis e Wiley Interscience.

- Descritores, obtenção de palavras-chave e seus descritores pelo Thesaurus.

- Diferença entre palavras-chave e descritores.

- Peer review.

- Navegação on-line numa base de dados.

- Open Access Library Journal.

- Agregadores de bibliotecas.

- Coleção on-line de ensino e aprendizagem, como o Merlot.

- C3. Usar com proficiência técnicas e serviços de apoio à pesquisa de informação científica relevantes para a área em estudo

- Operadores de pesquisa e sua utilização.

- Fator de impacto de publicações científicas, sua elaboração e obtenção por área do conhecimento.

- Really Simple Syndication - RSS.

- C4. Pesquisar com proficiência literatura cinzenta confiável na internet

- Literatura cinzenta.

- Repositório.

- C5. Utilizar adequadamente outras fontes de informação científica digital mais genéricas

- Google Acadêmico.

- Utilização do Referencial de Avaliação 
Durante o curso, após a clarificação dos conceitos, os estudantes do EM navegaram on-line com proficiência na recuperação de informação científica para o tema de estudo, na área de mecânica, considerado em cada grupo de estudantes. Cabe ressaltar que não se limitaram nos exemplos apresentados e tiveram competência para pesquisar em outras fontes de diversos formatos.

Para esclarecer o exposto, cita-se, como exemplo, um grupo de estudantes que pesquisou na área ferroviária. Durante o curso souberam direcionar e limitar a sua pesquisa, identificando como objetivo a resistência dos trilhos ferroviários e a identificação dos seus acessórios. Estabeleceram palavras-chave e descritores, o que proporcionou encontrar literatura sobre este assunto em bases de dados de bibliotecas e repositórios acadêmicos, evitando a sobrecarga de informação pelo uso de operados bolianos durante sua busca. Os estudantes sentiram-se confiantes ao recuperar informação sobre o seu projeto em periódicos fidedignos e também em fontes mais genéricas de vídeo.

Antes de aprenderem estas competências, este grupo de estudantes relatou que estava se sentindo "perdido" para realizar o projeto na área ferroviária, não sabendo como direcionar a pesquisa. Também lembrou que não estavam recuperando informação sobre Resistência dos Materiais e Elementos de Máquinas que atendesse o seu projeto.

A apresentaçaõ desta matéria durante o curso de extensão foi realizada segundo a apresentação das competências no Referencial de Avaliação. Inicialmente foi mostrado o critério $\mathrm{C} 1$, sobre a definição de uma necessidade de informação científica, evidenciando, na mesma ordem, os seus indicadores (do indicador I1 ao I3), explicando-os com os exemplos. Este procedimento de apresentação foi usado até o critério $C 5$, que trata da utilização adequada de fontes de informação científica consideradas genéricas, como o YouTube.

\section{Em Síntese}

Os resultados referentes à adaptação do Referencial de Avaliação de competências relacionadas com a LI, com recurso às TICs, do contexto de Ensino Superior (ES), na área de educação, para o Ensino Médio (EM), na área de mecânica, evidenciam que, basicamente, as alterações foram nos seus exemplos, com o intuito de clarificar a área de estudo considerada. Isso reflete que o referencial das competências de pesquisa de informação científica pode ser usado em qualquer área de investigação e níveis de aprendizagem, posto que, inicialmente, foi considerado o seu desenvolvimento a nivel do ES e, neste estudo, foi mostrada a sua utilização em âmbito do EM.

No que diz respeito à aquisição das competências consideradas por parte dos estudantes do EM durante o curso de extensão, esta pode ser justificada em razão de os mesmos estarem habituados à utilização de computadores portáteis, como tablet, para navegar na internet, e programas executados num sistema operacional em smartphones, não apresentando bloqueio na utilização das TIC. O mencionado vai ao encontro do contexto destes estudantes em relação às TICs apontadas por diversos autores, corroborando a ideia de que o Referencial de Avaliação dessas competências pode servir de base para o E/A da LI, inserido como ementa no currículo desta disciplina no âmbito do EM. 
Assim, pelo exposto, são apresentados os objetivos deste artigo, que se referem à apresentação das alterações e utilização do Referencial de Avaliação e a discussão destas alterações para atender o contexto dos estudantes do EM em relação aos uso das TICs.

\section{CONSIDERAÇÕES FINAIS}

O Referencial de Avaliação que se ocupa das competências relacionadas com a LI, com recurso às TICs, definido inicialmente na área de educação no âmbito do ES, pode ser usado em outras áreas e níveis de ensino. Considerando o seu uso em diversas áreas do conhecimento, esta assertiva deriva das alterações realizadas nos elementos dessa base de referência, que consistem nomeadamente nos exemplos para atender o curso ministrado na área de mecânica no EM. Relativamente à utilização do referencial em outros níveis de ensino, após a clarificação de alguns conceitos no decorrer do curso ministrado para os estudantes do EM, mostrado na análise dos resultados, foi observado um comportamento sem desembaraço, evidenciando a aquisição de competências na recuperação de informação científica da área estudada.

\section{Recomendação}

Deve-se ter em conta que para o E/A de LI apenas a leitura do Referencial de Avaliação não é suficiente para o seu entendimento por parte dos estudantes. É necessário esclarecer alguns conceitosAdaptações do Referencial de Avaliação. Trabalhos de pesquisa em âmbito do EM devem ser utilizados para a orientação desta matéria, preenchendo, dessa forma, a lacuna que existe nos estudantes em fase mais avançada, como os do ES.

Recomenda-se que não somente os estudantes estejam envolvidos na aprendizagem dessas competências, mas também os docentes e bibliotecários (JOVIC, 2016), pois a responsabilidade da escola é ajudar os estudantes a serem produtores do conhecimento refletido na aprendizagem (JOVIC, 2016; MARTINS DOS SANTOS FERREIRA; MOURÃO CASTIGLIONE, 2018; MCNICOL, 2015; PEREIRA; PINTO; MOURA, 2015).

\section{REFERÊNCIAS}

BUNDY, Alan. Australian and New Zealand Information Literacy Framework: principles, standards and practices. Austrália. 2004. Disponível em: http://www.anziil.org/index.htm. Acesso em: 5 jan. 2018.

DAVIDSON, Jeanne R.; MCMILLEN, Paula S.; MAUGHAN, Laurel S. Using the ACRL Information Literacy Competency Standards for Higher Education to Assess a University Library Instruction Program. Journal of Library Administration, Oregon, v. 36, n. 1-2, p. 97-121, 2002.

EISENBERG, Mike; JOHNSON, Doug; BERKOWITZ, Bob. Information, Communications, and Technology (ICT) Skills Curriculum Based on the Big6 Skills Approach to Information Problem-Solving. Library Media Connection, 2010. Disponível em: http://big6.com/media/freestuff/LMC_Big6-ICT_Curriculum_LMC_ MayJune2010.pdf. Acesso em: 29 abr. 2018.

ETS. ICT literacy assessment preliminary findings. New Jersey: Princeton, 2006.

FIGARI, Gérard. Évaluer: quel référentiel? Bruxelas: De Boeck-Wesmael, 1994.

GONZALEZ LOZANO, Gustavo et al. A review on design for manufacture of variable stiffness composite laminates. Proc IMechE Part B: J Engineering Manufacture, [s.I.], v. 230, n. 6, p. 981-992, 2016. Disponível em: http://journals.sagepub.com/doi/pdf/10.1177/0954405415600012. Acesso em: 30 abr. 2018.

HADJI, C. A avaliação, regras do jogo. Porto: Porto Editora, 1994. 
JOVIC, Marija. Understanding the Concept of Information Literacy Students - For Example, the City Library "Don Mihovil Pavlinovic" Imotski. Universal Journal of Educational Research, [s.I.], v. 4, n. 2, p. 378382, 2016. Disponível em: http://www.hrpub.org/journals/article_info.php?aid=3356. Acesso em: 15 out. 2017.

LE BOTERF, Guy. Construir as competências individuais e colectivas. Porto: ASA Editores, 2005.

LOPES, C. S.; GÜRDAL, Z.; CAMANHO, Pedro Ponces. Variable-stiffness composite panels: Buckling and firstply failure improvements over straight-fibre laminates. Computers \& Structures, [s.I.], v. 86, n. 9, p. 897907, 2008. Disponível em: https://www.sciencedirect.com/science/article/pii/S0045794907001654?via\%3Dihub. Acesso em: 30 abr. 2018.

LOPES, Carlos; PINTO, Maria. IL-HUMASS - Instrumento de avaliação de competências em literacia da informação: um estudo de adaptação à população portuguesa (Parte I). [S.I.], 2010. Disponível em: http://repositorio.ispa.pt/bitstream/10400.12/200/1/ILHUMASS\%25\%0A20\%25E2\%2580\%2593\%252 Oinstrumento\%2520de\%2520avalia\%25C3\%25A7\%25C3\%25A30\%2520\%252\%0A0de\%2520comptencias\%2520em\%2520literacia\%2520da\%2520informa\%25C3\%25A7\%25C3\%25A3o.pdf. Acesso em: 30 nov. 2010.

MARTIN, Coleen Meyers; GARCIA, Eric P.; MCPHEE, Marc. Education Libraries. Education Libraries, [s.I.], v. 35, n. 1-2, p. 34--47, Summer-Winter 2012. Disponível em: http://education.sla.org/wp-content/uploads/2012/12/35-1-2.pdf.

MARTINS DOS SANTOS FERREIRA, Giselle; MOURÃO CASTIGLIONE, Rafael Guilherme. TIC na educação: ambientes pessoais de aprendizagem nas perspectivas e práticas de jovens. Educ. Pesqui., São Paulo, v. 44, e153673, 2018,, p. 1-22, 2018. Disponível em: http://www.scielo.br/pdf/ep/v44/1517-9702ep-S1678-4634201702153673.pdf. Acesso em: 10 maio 2018.

MCNICOL, Sarah. Modelling information literacy for classrooms of the future. Journal of Librarianship and Information Science, [s.l.], v. 47, n. 474, p. 303-313, 2015. Disponível em: http://journals.sagepub.com/ doi/pdf/10.1177/0961000614526612. Acesso em: 30 abr. 2018.

PEREIRA, Sara; PINTO, Manuel; MOURA, Pedro. Níveis de literacia mediática: estudo exploratório com jovens do $12^{\circ}$ ano, 2015. Braga. Disponível em: http://www.lasics.uminho.pt/ojs/index.php/cecs_ebooks/ article/view/2117/2036. Acesso em: 28 dez. 2017.

PINTO, María; PONJUÁN, Gloria; URIBE-TIRADO, Alejandro. Conceptualización y perspectivas de la alfabetización informacional en Iberoamérica: un estudio Delphi. Information Research, Granada, v. 20, n. 3, 2015. Disponível em: http://www.webcitation.org/6blKgm8k7. Acesso em: 28 dez. 2017.

SALISBURY, Fiona; CORBIN, Jenny; PESETA, Tai. From cornerstone to capstone : information literacy collaboration across the curriculum collaboration across the curriculum. In: TRIENNIAL IATUL CONFERENCES, 34., 2013, Cape Town, África do Sul. Anais [...]. Cape Town, África do Sul. Disponível em: Cape Town, África do Sul. Acesso em: 27 out. 2013.

SORMUNEN, Eero; TANNI, Mikko; HEINSTRÖM, Jannica. Seeking information for a middle school history project: The concept of implicit knowledge in the students' transition from Kuhlthau's Stage 3 to Stage 4. Journal of the American Society for Information Science and Technology, Copenhague, v. 18, n. 3, p. 558573, 2013. Disponível em: http://doi.wiley.com/10.1002/asi.22786. Acesso em: 28 dez. 2017.

TIMMERS, Caroline F.; GLAS, Cees A. W. Developing scales for information seeking behaviour. Journal of Documentation, [s. I.], v. 66, n. 1, p. 46-69, 2010. Disponível em: http://www.emeraldinsight.com/ doi/10.1108/00220411011016362. Acesso em: 6 fev. 2011. 